\section{OPEN ACCESS}

Edited by:

Wei-Jun Qian

Pacific Northwest National Laboratory

(DOE), United States

Reviewed by:

Ashish K. Solanki,

Medical University of South Carolina,

United States

Hideki Yokoi,

Kyoto University Hospital, Japan

*Correspondence:

Kateryna Pierzynowska

katerina.goncharova@biol.lu.se

Danica Gruijic

dgrujic@allenapharma.com

Specialty section:

This article was submitted to

Nephrology,

a section of the journal

Frontiers in Medicine

Received: 02 July 2020 Accepted: 29 September 2020 Published: 24 November 2020

Citation:

Pierzynowska K, Deshpande A, Mosiichuk N, Terkeltaub R, Szczurek P, Salido E, Pierzynowski S and Grujic D

(2020) Oral Treatment With an

Engineered Uricase, ALLN-346,

Reduces Hyperuricemia, and Uricosuria in Urate Oxidase-Deficient

Mice. Front. Med. 7:569215.

doi: 10.3389/fmed.2020.569215

\title{
Oral Treatment With an Engineered Uricase, ALLN-346, Reduces Hyperuricemia, and Uricosuria in Urate Oxidase-Deficient Mice
}

\section{Kateryna Pierzynowska ${ }^{1,2,3 *}$, Aditi Deshpande ${ }^{4}$, Nadiia Mosiichuk ${ }^{5}$, Robert Terkeltaub ${ }^{6}$, Paulina Szczurek ${ }^{7}$, Eduardo Salido ${ }^{8}$, Stefan Pierzynowski ${ }^{2,3,9}$ and Danica Grujic ${ }^{4 *}$}

\footnotetext{
${ }^{1}$ Department of Animal Physiology, Kielanowski Institute of Animal Nutrition and Physiology Polish Academy of Sciences, Jabłonna, Poland, ${ }^{2}$ Department of Biology, Lund University, Lund, Sweden, ${ }^{3}$ SGP+Group, Trelleborg, Sweden, ${ }^{4}$ Allena Pharmaceuticals, Newton, MA, United States, ${ }^{5}$ Department of Biochemistry and Biotechnology, Vasyl Stefanyk Precarpathian National University, Ivano-Frankivsk, Ukraine, ${ }^{6}$ VA Medical Center, University of California, San Diego, La Jolla, CA, United States, ${ }^{7}$ Department of Animal Nutrition and Feed Sciences, National Research Institute of Animal Production, Balice, Poland, ${ }^{8}$ Hospital Universitario de Canarias, Universidad La Laguna \& Center for Rare Diseases (CIBERER), Tenerife, Spain, ${ }^{9}$ Department of Biology, Institute Rural Medicine, Lublin, Poland
}

Limitations in efficacy and/or tolerance of currently available urate-lowering therapies (ULTs), such as oral xanthine oxidase inhibitors, uricosurics, and intravenous uricase agents contribute to the development of refractory gout. Renal excretion is the major route of uric acid elimination, but the intestinal tract plays an increasingly recognized role in urate homeostasis, particularly in chronic kidney disease (CKD) in which the renal elimination of urate is impaired. We targeted intestinal degradation of urate in vivo with ALLN-346, an orally administered, engineered urate oxidase, optimized for proteolytic stability, and activity in the gut. We tested ALLN-346 in uricase/urate oxidase deficient mice (URKO mice) with severe hyperuricemia, hyperuricosuria, and uric acid crystalline obstructive nephropathy. A total of 55 male and female URKO mice were used in the two consecutive studies. These seminal, proof-of-concept studies aimed to explore both short- (7-day) and long-term (19-day) effects of ALLN-346 on the reduction of plasma and urine urate. In both the 7-and 19-day studies, ALLN-346 oral therapy resulted in the normalization of urine uric acid excretion and a significant reduction of hyperuricemia by 44 and 28\% when therapy was given with food over $24 \mathrm{~h}$ or was limited for up to $6 \mathrm{~h}$, respectively. Fractional excretion of uric acid (FEUA) was normalized with ALLN-346 therapy. Oral enzyme therapy with engineered urate oxidase (ALLN-346) designed to degrade urate in the intestinal tract has the potential to reduce hyperuricemia and the renal burden of filtered urate in patients with hyperuricemia and gout with and without CKD.

Keywords: gout, CKD, urolithiasis, urate-lowering therapy, ABCG2 


\section{INTRODUCTION}

Hyperuricemia, defined as a serum urate (sUA) concentration greater than either 6.8 or $7.0 \mathrm{mg} / \mathrm{dl}$ (1), affects around $20 \%$ of adults within the United States (2) and promotes the development of gout, which in the United States has a prevalence of $\sim 4 \%$. In gout, tissue deposits of monosodium urate crystals promote acute and chronic inflammatory arthritis (3). In addition, urolithiasis is common in gout and is promoted by decreased solubility of uric acid in the acidic $\mathrm{pH}$ of the urine and increased urinary uric acid concentration (4). Gout is associated with a decreased health-related quality of life and increased health care utilization (5). In addition, hyperuricemia has been identified as a potential contributor to the pathophysiology of multiple comorbidities associated not only with gout, but also with asymptomatic hyperuricemia. These include hypertension, chronic kidney disease (CKD), obesity, metabolic syndrome, type 2 diabetes, and coronary artery disease (6-9).

Although some gout patients have heritable conditions that cause the overproduction of uric acid (10), insufficient renal excretion of uric acid is by far the most frequent cause of hyperuricemia in gout (11). In healthy subjects, approximately two thirds of uric acid excretion is renal $(12,13)$. Urate is also secreted into the small intestine, where it can be degraded by gut bacteria (intestinal uricolysis) or eliminated by defecation $(12,14)$. In individuals with normal uric acid metabolism, approximately one third ( $\sim 200 \mathrm{mg} /$ day) of daily urate elimination occurs by intestinal secretion $(12,13)$ with the ability to increase urate elimination to $\sim 300 \mathrm{mg} /$ day.

In those with CKD, the fraction of urate eliminated intestinally may increase to between 50 and $70 \%$, surpassing renal elimination $(12,15,16)$. The importance of intestinal elimination of urate in patients with CKD has been supported by the observation that sevelamer, a non-absorbed phosphate binder, which is also a non-selective urate binder, significantly reduces sUA concentration in hyperuricemic patients on hemodialysis $(17,18)$. However, the magnitude of the sevelamer urate-lowering effects was only moderate, achieving a mean sUA reduction of $\sim 0.6-0.7 \mathrm{mg} / \mathrm{dl}$ in a randomized clinical trial (17) with the highest sUA reduction observed in subjects with the most severe hyperuricemia.

Urate transport and the maintenance of urate homeostasis involves a complex network of transporters located in the kidneys and the intestine (19). Major transporters involved in renal urate disposition that are encoded by gout-risk genes include SLC22A12 (encoding URAT1), SLC2A9 (encoding Glut9), and the urate secretory transporter ABCG2 (ATP-binding cassette) (13). In contrast to the majority of renal urate transporters, $A B C G 2$ is also expressed in the small intestine (20), where it plays an important role in lowering sUA (21). Moreover, the specific impact of intestinal $A B C G 2$ on urate homeostasis appears to increase as renal function declines $(15,16)$. This is also illustrated in animal models in which normal sUA levels were maintained with increased intestinal $A B C G 2$ expression in healthy rats subjected to subtotal nephrectomy (22). Similarly, decreased intestinal urate elimination and hyperuricemia development (23) resulted from the knockout of the $A B C G 2$ transporter ( $A b c g 2$ null mice). In humans, certain dysfunctional $A B C G 2$ variants are associated with hyperuricemia and increased risk of gout, including the early onset of gout disease and expression of the severe tophaceous gout phenotype (24). Such variants include the widely prevalent $A B C G 2$ Q141K (rs2231142), which has a particularly high allele frequency (up to $\sim 30 \%$ ) in multiple East Asian populations at large and, not unexpectedly, an even higher allele frequency (up to $\sim 50 \%$ ) in gout patients from those populations (25). Severe impairment of ABCG2 function causes hyperuricemia and gout associated with excessive renal urate filtration ("renal uric acid overload") (24).

Hyperuricemia and gout are prevalent in patients with CKD relative to those with preserved renal function with the estimated prevalence of gout in patients with stage 3 CKD in the United States being between $\sim 20$ and 30\% (2). This poses a unique challenge to clinicians seeking effective treatment for patients with CKD as both renal impairment and urolithiasis limits the use of existing urate-lowering therapies (ULTs), including uricosurics that increase uric acid excretion and also xanthine oxidase inhibitors due to increased risk of potential toxicity with the optimal therapeutic dose $(26,27)$. Taking into account the limitations of present therapies and the robust secretion of urate into the small intestine $(12,14)$, a new uricase, ALLN-346, was designed as a potentially safer alternative therapy that is not absorbed into circulation and has the potential to degrade the urate secreted and/or formed in the small intestine, thereby reducing the systemic urate burden. ALLN-346 was designed to achieve this outcome through the protein engineering of Candida utilis uricase to impart proteolytic stability while not decreasing the specific activity compared to the wild-type enzyme using proprietary ProteinGPS technology (Atum, CA, USA). Through a machine learningguided molecular evolution process, the proteolytic stability of the uricase in the intestinal tract was enhanced, enabling it to degrade urate (U.S. Patent Application \#20200071681).

The gene encoding uricase/urate oxidase ( $\mathrm{UrOx})$ was inactivated during evolution in humans, and to assess the efficacy of ALLN-346 in a "human-like" model of hyperuricemia, the UrOx knockout mouse model was used. The B6;129S7Uoxtm1Bay/J mouse strain (URKO mice) (28) is characterized by severe hyperuricemia with an average plasma UA of 11.0 $\pm 1.7 \mathrm{mg} / \mathrm{dl}$, which is $\sim 12$ times higher than that in the wild-type controls $(0.9 \pm 0.3 \mathrm{mg} / \mathrm{dl})$ with active uricase liver enzymes. These mice have pronounced hyperuricosuria, and they spontaneously develop uric acid crystalline obstructive nephropathy (28). Our work demonstrates that oral treatment with ALLN-346 reduces both hyperuricemia and hyperuricosuria in URKO mice via effective degradation of urate secreted in the intestinal tract.

\section{MATERIALS AND METHODS}

Experiments were performed in accordance with the recommendations in the Guide for the Care and Use of Laboratory Animals of the National Institutes of Health. All experimental procedures were approved by the University 
of Lund Ethics Review Committee on Animal Experiments (Approval No. M14331-17). All efforts were made to minimize animal suffering during experimental procedures.

\section{Molecular Evolution of Candida utilis Uricase}

Uricase mutants were developed using protein-engineering principles by a synthetic biology company ATUM (CA, USA). Briefly, DNA fragments encoding the 95 mutant $C$. utilis uricases were cloned into a rhamanose pD861-NH expression vector with an N-terminal His-tag. Following expression in Escherichia coli cells, recombinant mutants were purified using an Ni-NTA column, according to standard procedures and further tested for enzymatic activity and stability in the presence of pancreatin (Sigma-Aldrich, Cat No. P7545). Briefly, $25 \mathrm{ng} / \mu \mathrm{l}$ of uricase was incubated with $20 \mathrm{ng} / \mu \mathrm{l}$ of pancreatin at $37^{\circ} \mathrm{C}$ for up to $200 \mathrm{~min}$ in simulated intestinal fluid buffer $(50 \mathrm{mM}$ potassium phosphate, $\mathrm{pH}$ 6.8) in a 96-well plate. Following incubation with pancreatin (Cat No. P7545, Sigma-Aldrich, St. Louis, MO, USA) for the indicated time points, the best mutants were selected based on the enzymatic activity using an absorption-based assay (enzymatic oxidation of uric acid to 5-hydroxyisourate by uricase results in a corresponding drop in $293 \mathrm{~nm}$ absorbance over time). The DNA and amino sequence of ALLN-346 chosen for further development is shown in the U.S. recombinant uricase enzyme patent application \#20200071681.

The novel engineered uricase is the active compound of ALLN-346 with an optimal $\mathrm{pH}$ activity range of between 6.5 and 9; the enzyme is acid sensitive and is irreversibly inactivated below a $\mathrm{pH}$ of 5 . Hence, in order to protect the uricase enzyme from the acidic $\mathrm{pH}$ of the stomach, ALLN-346 was administered as a food/enzyme mix together with $1 \%$ bicarbonate $(\mathrm{w} / \mathrm{w})$ as a basifier.

\section{Animals}

A total of 55 male and female mice (strain: B6;129S7Uoxtm1Bay/J) generated at Jackson Laboratory (USA) (https:// www.jax.org/strain/002223) were used in the study. When the study was initiated, the mice were $>6$ weeks of age with a body weight of $\sim 22-26 \mathrm{~g}$. Mice were maintained on a 12-h day/night cycle with lights on from 7:30 a.m.-7:30 p.m. (07:30-19:30 h) and dark from 7:30 p.m.-7:30 a.m. (19:30-07:30 h), respectively. Prior to the experimental period, the mice were housed in groups in regular cages equipped with water bottles and feeders (6 mice/cage) with beta chip bedding. Allopurinol, which was needed to ensure mouse survival, was added to their drinking water at a dose of $150 \mathrm{mg} / \mathrm{L}$ (28). Following randomization into groups, the mice were individually housed in collection cages, equipped with a small feeding bowl and a drinking bottle. To label the mice, each cage was clearly identified with a color-coded card indicating the study number, animal number, sex, and group allocation. A standard certified commercial diet (standard rodent pelleted feed; Lactamin, Vadstena, Sweden) was provided ad libitum during the entire study period, including the adaptation period, during which mice were acclimatized to the collection cages. Drinking water was provided ad libitum as well. Animal feed intake during treatment was measured daily, and their body weight and water intake were measured weekly.

\section{Experimental Design and ALLN-346 Dosing}

The experimental design comprised two consecutive studies, a short- (7-day) and a long-term (19-day) study. Both studies had a 7-day-long pretreatment period when allopurinol was withdrawn from the drinking water to induce hyperuricemia and hyperuricosuria. During this time, mice were also adapted to collection cages and to the special feed containing ALLN346. In both studies, mice were randomized into groups based on plasma urate concentrations, measured from a single blood sample collected at the end of the pretreatment period irrespective of gender. The targeted daily dose of ALLN-346 was $\sim 2,000$ units/day.

In the initial 7-day study, 31 female and male mice with an average body weight of $26.3 \pm 0.69 \mathrm{~g}$ were divided into four groups: control ( $n=7,3$ male, 4 female), in which no treatment was given; ALLN-346 ( $n=7,5$ male, 2 female), in which mice were given ALLN-346 mixed with food that was enriched with $1 \%$ sodium bicarbonate (basifier); and two allopurinol groups, ALLO $50(n=8,3$ male, 5 female $)$ and ALLO $150(n=9$, 4 male, 5 female), in which mice were treated with allopurinol at doses of 50 and $150 \mathrm{mg} / \mathrm{l}$ provided in their drinking water (28), respectively, and were also used as a comparator to the ALLN-346 group. The daily dose of ALLN-346 was calculated based on average daily food consumption. The ALLN-346 was administered in feeders during the 24-h cycle in the form of a "cake" prepared from regular chow powder rodent diet that was enriched with peanut butter and olive oil to provide a uniform food/enzyme mix. The food cake mix contained $100 \mathrm{~g}$ chow powder rodent diet, $50 \mathrm{~g}$ peanut butter, and $\sim 12-25 \mathrm{~g}$ olive oil and was provided ad libitum. Adaptation to this diet without ALLN-346 addition occurred during the pretreatment period.

In the second, 19-day study, 24 female and male mice with an average body weight of $22.0 \pm 2.89 \mathrm{~g}$ were divided into three groups: control ( $n=7,4$ male, 3 female), no treatment; ALLO 90 ( $n=7,5$ male, 2 female), in which mice were treated with allopurinol (90 mg/l in drinking water); and ALLN-346 ( $n=10$, 7 male and 3 female), in which mice were treated with ALLN346 mixed with food enriched with $1 \%$ sodium bicarbonate as a basifier. ALLN-346 was offered as a "single feed" during 4-6h at night in the form of a "milk-fat cake" consisting of a mixture of $\sim 130 \mathrm{~g}$ of dry milk, $\sim 30 \mathrm{~g}$ butter, $75 \mathrm{~g}$ cornstarch, $75 \mathrm{~g}$ corn powder, and $\sim 80 \mathrm{~g}$ water mixed. The mice were adapted to this diet during the pretreatment period.

\section{Blood Collection}

Blood samples $(\sim 50 \mu \mathrm{l})$ were collected from the submandibular vein into Microvette ${ }^{\circledR} 100 \mathrm{LH}$ tubes (SARSTEDT AG\&Co. KG, Numbrecht, Germany). In general, blood samples were collected at the end of the pretreatment, treatment, and follow-up periods. After collection, blood samples were centrifuged at $3,000 \mathrm{~g}$ at $4^{\circ} \mathrm{C}$ for $10 \mathrm{~min}$ and analyzed fresh or stored for up to $48 \mathrm{~h}$ at $4^{\circ} \mathrm{C}$ until analysis. 


\section{Urine Collection}

A $24 \mathrm{~h}$ urine sample was collected into collection tubes 3 times during the pretreatment and 3 times during the treatment period. To prevent precipitation of salts of uric acid, 1-2 drops of $\mathrm{NaOH}$ $(8 \mathrm{M})$ were added to the collection tubes. Urine samples were analyzed fresh or stored for up to $48 \mathrm{~h}$ at $4^{\circ} \mathrm{C}$ until analysis.

\section{Urate and Creatinine Measurement in Plasma, Urine, and Chyme}

The concentration of urate in plasma and urine samples was measured spectrophotometrically, using an enzymatic Uric Acid Assay Kit Liquick Cor-UA 60 PLUS (cat. \# 2-258, P.Z. Cormay S.A., Lomianki, Poland), according to the manufacturer's protocol. Before measurements, urine samples were diluted 5-15 times with distilled water, depending on the experimental group; the plasma samples were analyzed without dilution.

Urate analysis was also performed in chyme samples collected from the stomach, small intestine (duodenum, jejunum, and ileum), and colon of the mice at the end of the 19-day study. Samples were extracted with $0.068 \mathrm{M} \mathrm{Li} 2 \mathrm{CO} 3(\mathrm{pH}=$ 11.5) (1:3, weight: volume) and, following gentle mixing, were incubated in boiling water for $10 \mathrm{~min}$, followed by $15 \mathrm{~min}$ centrifugation at $13,000 \mathrm{~g}$ at $4{ }^{\circ} \mathrm{C}$. The resultant supernatant was then filtered through the Centrifugal Concentrator Vivaspin 500, $10 \mathrm{kDa}$ (Vivaspin ${ }^{\circledR}$, Sartorius, Göttingen, Germany) at $15 \mathrm{~min}$ at $13,000 \mathrm{~g}$ and $4^{\circ} \mathrm{C}$. The filtrate, with or without dilution, was used to determine urate concentrations, using the same kit as that used for urine and plasma urate determinations.

The concentration of creatinine in the urine and plasma was measured spectrophotometrically using a colorimetric creatinine assay kit (ab204537, Abcam, Cambridge, UK) and an enzymatic Creatinine Assay Kit Liquick Cor-CREA ENZYMATIC 60 (cat. \# 2-267, P.Z. Cormay S.A., Lomianki, Poland), respectively, in accordance with the manufacturer's instructions.

\section{Calculation of Renal Test Parameters}

Fractional excretion of urate (FEUA) as a ratio of creatinine and urate clearance, was calculated as follows:

$$
\begin{aligned}
& \text { FEUA (\%) } \\
& =\frac{\text { Urinary uric acid }\left(\frac{m g}{d l}\right) \times \text { Serum creatinine }\left(\frac{m g}{d l}\right)}{\text { Serum uric acid }\left(\frac{m g}{d l}\right) \times \text { Urinary creatinine }\left(\frac{m g}{d l}\right)} \times 100
\end{aligned}
$$

\section{Statistical Analysis}

Statistical analysis was performed using a repeated-measures ANOVA with the Geisser-Greenhouse correction followed by a Bonferroni correction for multiple comparisons. All analyses were carried out using Prism, version 8 (GraphPad Software, Inc., San Diego, CA, USA). Data are expressed as mean \pm standard error of the mean (SEM). The distribution of the parameters was checked using a Shapiro-Wilk normality test. In all statistical analyses, $p<0.05$ was considered significant.

\section{RESULTS}

\section{Engineering Urate Oxidase Design and Selection}

An analysis of pancreatin stability of 196 recombinant mutant C. utilis uricases generated during two rounds of molecular evolution was used to identify key substitutions that contribute to improved pancreatin stability. Results for C. utilis uricase mutants with the most improved pancreatin stability were confirmed over multiple protein preparations. Engineered UrOx was identified as the enzyme with 20 -fold increased stability against pancreatin compared to the wild-type C. utilis uricase and without significantly decreased specific activity. Engineered UrOx exhibited a half-life of $85.3 \mathrm{~min}$ compared to $4.3 \mathrm{~min}$ for the wild-type $C$. utilis enzyme at $80 \mathrm{ng} / \mu \mathrm{l}$ soluble pancreatin.

\section{The 7-Day Study}

Removal of allopurinol from the drinking water in the 7-day study resulted in severe hyperuricemia and hyperuricosuria with mean values of plasma and urine urate of $14.01 \pm 0.86$ $\mathrm{mg} / \mathrm{dl}$ and $5.03 \pm 0.35 \mathrm{mg} / 24 \mathrm{~h}$, respectively. Hyperuricemia was significantly reduced by $51 \%$ (from $13.20 \pm 2.63$ to $6.50 \pm 1.10$ $\mathrm{mg} / \mathrm{dl}$ ), $44 \%$ (from $14.50 \pm 0.90$ to $8.11 \pm 0.53 \mathrm{mg} / \mathrm{dl}$ ), and $69 \%$ (from $13.81 \pm 1.73$ to $4.31 \pm 0.59 \mathrm{mg} / \mathrm{dl}$ ) in the ALLO 50, ALLN346 , and ALLO 150 groups, respectively $(p<0.05)$. No changes in plasma UA concentrations were observed in the control group (Figure 1A).

Daily urate excretion was significantly reduced by $35 \%$ (from $5.01 \pm 0.25$ to $3.24 \pm 0.50 \mathrm{mg} / 24 \mathrm{~h}$ ), $87 \%$ (from $4.85 \pm 0.85$ to $0.63 \pm 0.15 \mathrm{mg} / 24 \mathrm{~h}$ ), and $66 \%$ (from $6.38 \pm 0.65$ to $2.20 \pm 0.41$ $\mathrm{mg} / 24 \mathrm{~h}$ ) from pretreatment in the ALLO 50, ALLN-346, and ALLO 150 groups, respectively $(p<0.05)$. No changes in daily urate excretion were observed in the control group (Figure 1B).

Pre-randomization data for food and water intake and daily urine volumes are provided in Table 1. Food intake during the treatment period was similar between the experimental groups; however, it was significantly higher when compared to the intake during the pretreatment period $(p<0.05)$. The mean water intake during the pretreatment period was $11.93 \pm 0.75 \mathrm{ml} / 24 \mathrm{~h}$ (Table 1). Although water consumption remained stable in the control and ALLN-346 groups during treatment, a significant ( $p$ $<0.05$ ) decrease in water intake was observed in the ALLO 50 and ALLO 150 groups (Table 1). The mean urine volume during the pretreatment period was $7.45 \pm 1.01 \mathrm{ml} / 24 \mathrm{~h}$ (Table 1), and during treatment, an almost 2 -fold decrease in daily urine volume was observed in the control, ALLO 50, and ALLO 150 groups ( $p$ $<0.05$ ) although no changes in urine volume were observed in the ALLN-346 group.

\section{The 19-Day Study}

Similar to the 7-day study, removal of allopurinol from the drinking water for a week prior to randomization resulted in the development of severe hyperuricemia and hyperuricosuria in mice with mean plasma and urine urate levels of $12.93 \pm$ $0.72 \mathrm{mg} / \mathrm{dl}$ and $6.79 \pm 0.24 \mathrm{mg} / 24 \mathrm{~h}$, respectively. Plasma UA concentrations were significantly decreased $(p<0.05)$ by $62 \%$ (from $12.92 \pm 1.92$ to $4.93 \pm 0.90 \mathrm{mg} / \mathrm{dl}$ ) and $28 \%$ (from $12.96 \pm$ 

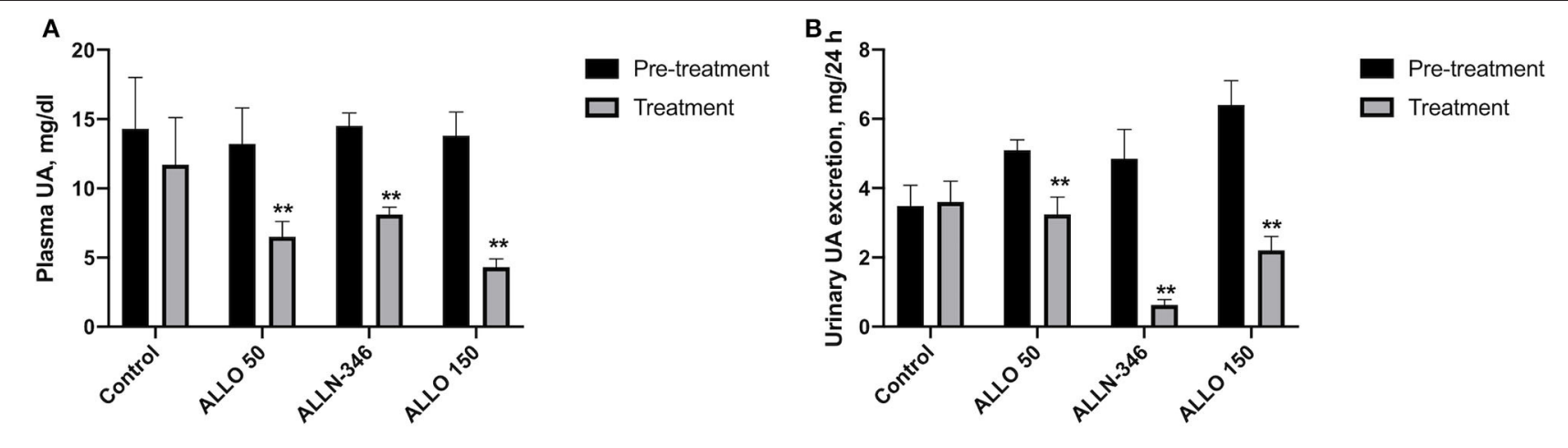

FIGURE 1 | Plasma UA level (A) and urinary UA excretion (B) in uricase/UrOx deficient mice following the 7-day study. Data are shown as mean \pm SEM. Control group, no treatment $(n=7)$; ALLO 50 group, allopurinol supplemented in drinking water (50 mg/L) $(n=8)$; ALLO 150 group, allopurinol supplemented in drinking water (150 mg/L) ( $n=9$ ); ALLN-346 group, ALLN-346 mixed with food provided ad libitum $(n=7) .{ }^{*}$ Statistically significant differences considering $p<0.05$ for comparison between pretreatment and treatment periods.

TABLE 1 | Pre- and post-treatment daily food and water intake as well as urine output in uricase/UrOx deficient mice for both the 7-and 19-day studies.

\begin{tabular}{|c|c|c|c|c|c|c|}
\hline Groups & \multicolumn{2}{|c|}{ Food intake (g) } & \multicolumn{2}{|c|}{ Water intake (ml) } & \multicolumn{2}{|c|}{$24 \mathrm{~h}$ urine volume $(\mathrm{ml})$} \\
\hline \multicolumn{7}{|c|}{ 7-day study } \\
\hline Control & \multirow{3}{*}{$2.54 \pm 0.17^{a}$} & $3.20 \pm 0.10^{b}$ & \multirow{3}{*}{$11.93 \pm 0.75^{a}$} & $10.60 \pm 1.19^{a, b}$ & \multirow{3}{*}{$7.45 \pm 1.01^{\mathrm{a}}$} & $4.30 \pm 0.55^{b}$ \\
\hline ALLO 50 & & $3.10 \pm 0.07^{b}$ & & $7.30 \pm 0.66^{b}$ & & $4.20 \pm 0.53^{b}$ \\
\hline ALLO 150 & & $3.20 \pm 0.11^{b}$ & & $7.90 \pm 0.48^{b}$ & & $4.10 \pm 0.24^{b}$ \\
\hline \multicolumn{7}{|c|}{ 19-day study } \\
\hline Control & \multirow{3}{*}{$3.80 \pm 0.08$} & $3.85 \pm 0.16$ & \multirow{3}{*}{$14.59 \pm 0.52^{\mathrm{a}}$} & $17.26 \pm 1.35^{\mathrm{a}}$ & \multirow{3}{*}{$7.87 \pm 0.53^{\mathrm{a}}$} & $11.12 \pm 1.72^{\mathrm{a}, \mathrm{b}}$ \\
\hline ALLO 90 & & $3.78 \pm 0.18$ & & $14.73 \pm 0.65^{\mathrm{a}}$ & & $8.17 \pm 1.12^{a, b}$ \\
\hline ALLN-346 & & $3.57 \pm 0.13$ & & $18.49 \pm 1.07^{b}$ & & $12.75 \pm 1.09^{b}$ \\
\hline
\end{tabular}

Data are shown as mean \pm SEM. Control group, no treatment $(n=7)$; ALLO 50 group, allopurinol supplemented in drinking water $(50 \mathrm{mg} / L)(n=8)$; ALLO 150 group, allopurinol supplemented in drinking water (150 mg/L) ( $n=9$ ); ALLN-346 group, ALLN-346 mixed with food provided ad libitum $(n=7)$. The different superscript letters describe statistically significant differences when $p<0.05$, pre- vs. post-treatment.

0.78 to $9.30 \pm 0.70 \mathrm{mg} / \mathrm{dl}$ ) in the ALLO 90 and ALLN-346 groups, respectively. No significant changes in plasma UA concentrations were observed in the control group (Figure 2A).

Urine urate excretion was significantly decreased $(p<$ 0.05 ) by $72 \%$ (from $6.45 \pm 0.38$ to $1.82 \pm 0.34 \mathrm{mg} / 24 \mathrm{~h}$ ) and $88 \%$ (from $6.62 \pm 0.31$ to $0.82 \pm 0.10 \mathrm{mg} / 24 \mathrm{~h}$ ) in the ALLO 90 and ALLN-346 groups, respectively. Interestingly, a reduction in urine urate excretion of $46 \%$ (from $7.36 \pm 0.58$ to $3.97 \pm 0.5 \mathrm{mg} / 24 \mathrm{~h}$ ) was also observed in the control group $(p<0.05)$ (Figure 2B).

Declining renal function was observed during the 19-day treatment period with UA clearance significantly reduced in all experimental groups $(p<0.05)$ (Table 2), which is not unexpected in this mouse strain, which requires treatment with high doses of allopurinol for survival. Pretreatment plasma creatinine concentrations ranged between 0.30 and $0.34 \mathrm{mg} / \mathrm{dl}$ and, following randomization, significantly increased $(p<0.05)$ in all study groups (Table 2). This was reflected in the reduction of FEUA by 40 and $87 \%$ in the control and ALLN-346 groups, respectively $(p<0.05)$ during the 19-day treatment period
(Table 2). No changes in FEUA were observed in the ALLO 90 group (Table 2).

The mean range of urate in the chyme collected from the stomach and colon of the uricase/UrOx deficient mice was between 0.04 and $0.08 \mathrm{mg} / \mathrm{g}$ based on wet weight of digesta and was similar between groups. However, in the small intestine, the urate levels were several fold higher with the highest measured urate concentration observed in the control group $(610 \pm 0.060$ $\mathrm{mg} / \mathrm{g}$ wet weight), and lower levels of urate, by 29 and 55\%, were recorded in the ALLN-346 and ALLO groups, respectively (Figure 3).

The food and water intake as well as daily urine volume are shown in Table 1. The food intake ranged between 3.6 and $4.0 \mathrm{~g} /$ day and was not significantly different between groups during both study periods. The water intake during the pretreatment period ranged between 13.5 and $16.0 \mathrm{~mL} /$ day and was not significantly different during the treatment period with the exception of the ALLN-346 group, in which it increased significantly by $27 \%(p<0.05)$, compared to pretreatment values and that observed in the other study groups. Accordingly, a 

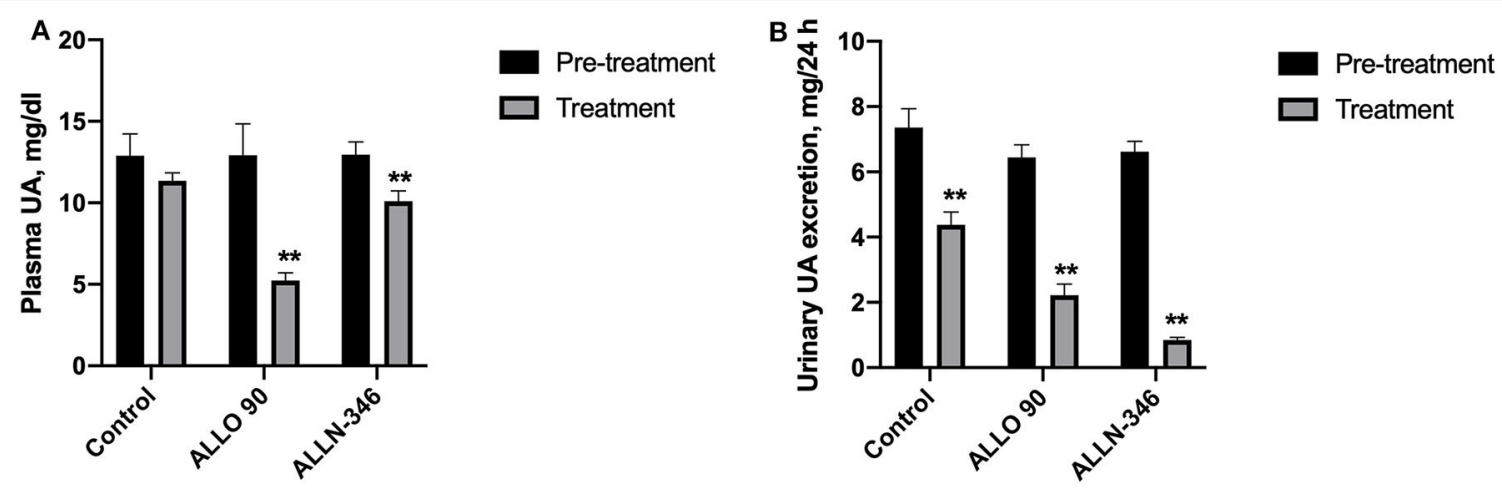

FIGURE 2 | Plasma UA level (A) and urinary UA excretion (B) in uricase/UrOx deficient mice following the 19-day study. Data are shown as mean \pm SEM. Control group, no treatment ( $n=7$ ); ALLO 90 group, allopurinol supplemented in drinking water (90 mg/L) ( $n=7$ ); ALLN-346 ( $n=10)$, ALLN-346 mixed with food and provided for up to $6 \mathrm{~h}$. ${ }^{*}$ Statistically significant differences considering $p<0.05$ for comparison between pretreatment and treatment periods.

TABLE 2 | Pre- and post-treatment renal test parameters in uricase/UrOx deficient mice in the 19-day study.

\begin{tabular}{|c|c|c|c|c|c|c|c|c|}
\hline \multirow[t]{2}{*}{ Group } & \multicolumn{2}{|c|}{ Plasma creatinine (mg/dL) } & \multicolumn{2}{|c|}{ UA clearance (mL/h) } & \multicolumn{2}{|c|}{ Creatinine clearance $(\mathrm{ml} / \mathrm{h})$} & \multicolumn{2}{|c|}{ FEUA (\%) } \\
\hline & Pre-treatment & Treatment & Pre-treatment & Treatment & Pre-treatment & Treatment & Pre-treatment & Treatment \\
\hline Control & $0.34 \pm 0.05$ & $0.43 \pm 0.05^{\star}$ & $2.58 \pm 0.31$ & $1.53 \pm 0.17^{\star}$ & $9.99 \pm 2.06$ & $10.32 \pm 2.45$ & $29.94 \pm 4.91$ & $18.0 \pm 2.39^{*}$ \\
\hline ALLO 90 & $0.30 \pm 0.06$ & $0.49 \pm 0.08^{\star}$ & $2.43 \pm 0.36$ & $1.54 \pm 0.24^{\star}$ & $14.3 \pm 3.27$ & $11.57 \pm 3.42$ & $19.56 \pm 2.24$ & $19.90 \pm 5.1$ \\
\hline ALLN-346 & $0.32 \pm 0.03$ & $0.42 \pm 0.03^{\star}$ & $2.38 \pm 0.24$ & $0.39 \pm 0.07^{\star}$ & $10.16 \pm 1.32$ & $12.15 \pm 1.70$ & $26.54 \pm 3.52$ & $3.46 \pm 0.58^{*}$ \\
\hline
\end{tabular}

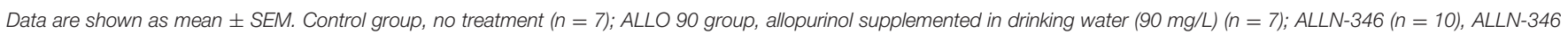
mixed with food and provided for up to $6 \mathrm{~h}$. ${ }^{*}$ Describes statistically significant differences within groups when $p<0.05$, pre- vs. post-treatment.

significantly increased daily urine production was observed in the ALLN-346 group following the 19-day treatment period compared to pretreatment values $(p<0.05)$ (Table 1).

\section{DISCUSSION}

The prevalence of hyperuricemia and gout has increased in recent years, and there is an unmet need for new therapies that help patients with refractory gout or CKD. We tested a potential new therapeutic option for patients with hyperuricemia and gout in URKO mice that mimics, to an extent, the disease in subjects with severe hyperuricemia and kidney impairment. The two current studies described were designed as seminal, proof-of-concept, preclinical investigations prior to initiating early clinical trials. Both the 7- and 19-day studies demonstrate that oral enzyme therapy with ALLN-346 resulted in a substantial reduction in the body urate burden as evident in the normalization of urate excretion and a significant reduction in plasma urate concentration. In the 7-day study, the mean reduction in plasma urate concentration was $44 \%$, which was similar to the reduction achieved with the allopurinol dose of $50 \mathrm{mg} / \mathrm{l}$. However, this reduction was higher in comparison to the mean reduction of $28 \%$ observed during the 19 -day study. Because the daily ALLN346 dose was similar between studies, $\sim 2,000$ units/day, the difference in the reduction of plasma urate observed was likely due to the dosing frequency of the ALLN-346. In the 7-day study, the ALLN-346 was provided with food ad libitum, throughout the entire day, although access to ALLN-346 in the 19-day study

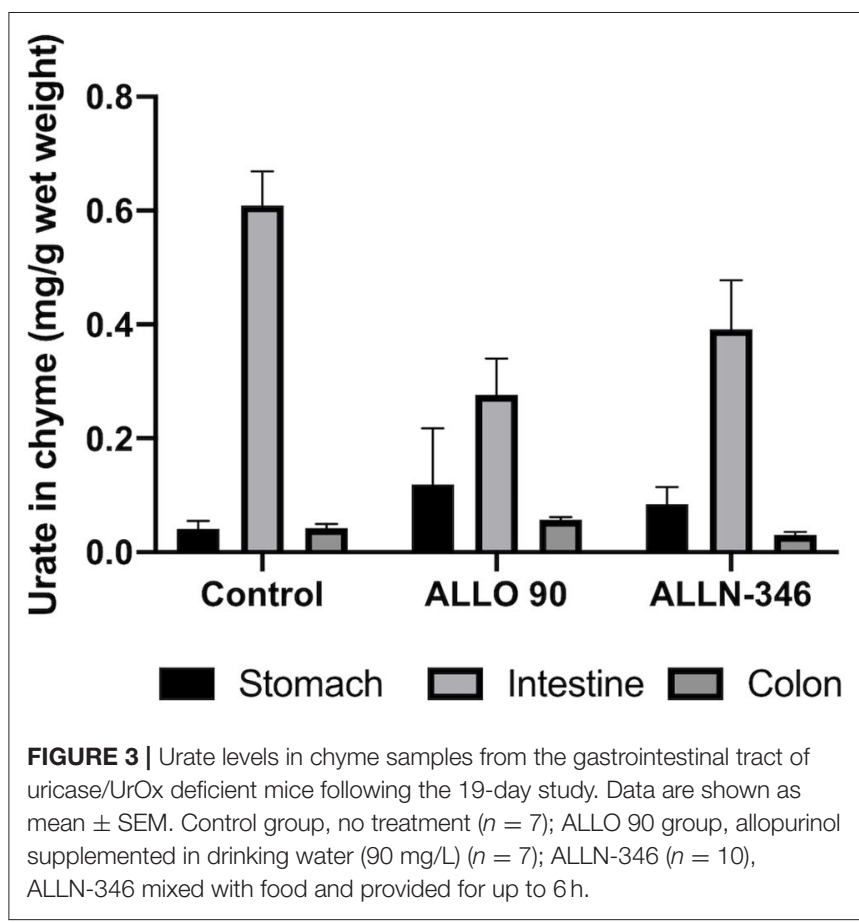

was restricted toa 4 - to 6 -h window at night, mimicking, to an extent, the effect of a single daily dose. Given our understanding of gut urate homeostasis $(12,29,30)$, our findings suggest that 
secretion of UA in the intestine likely occurs over the entire day in the setting of severe hyperuricemia and impaired renal function.

Reduction in renal UA burden and excretion by ALLN-346 was a central finding in this seminal study. As demonstrated in both studies, UA excretion was normalized with the use of ALLN346 or allopurinol. In these treatment groups, the normalization of UA excretion was rapid. Interestingly, the reduction in uric acid excretion in both the ALLO 50 and ALLO 150 groups was lower than that in the ALLN-346 group (88\% in ALLN346 group vs. $72 \%$ in ALLO group, $p=0.0198$ ). We speculate that this difference is either a result of the dose of allopurinol or possible off-target effects of allopurinol. The major active allopurinol metabolite, oxypurinol, partially blocks the URAT1 transporter. By blocking URAT1, urate reabsorption from the renal proximal tubules is altered with a net result of increased urate excretion (11). In contrast, ALLN-346 is expected to degrade urate only within the intestine, including both the urate that is secreted into the intestine via extrarenal elimination and the urate formed in the intestine itself. Consequently, ALLN346 is expected to affect the urate equilibrium across different compartments in the body (kidney, joints, blood, and intestine) and thereby reduce overall hyperuricemia. Thus, in contrast to other available ULTs, ALLN-346 does not directly affect the generation of urate within the body or function of different urate transporters in kidneys/intestine but rather degrades urate within the intestinal tract.

In the 19-day study, a significant increase in plasma creatinine was observed at the end of the study in all experimental groups. These results are not in agreement with previously published work by $\mathrm{Lu}$ et al. (31), in which after 2 weeks of allopurinol supplementation, at doses of either $40 \mathrm{mg} / \mathrm{kg}$ or $100 \mathrm{mg} / \mathrm{kg}$, serum creatinine was reduced in comparison to the control, untreated group. Mean consumption of allopurinol in the current study was $57.4 \mathrm{mg} / \mathrm{kg}$ body weight and, thus, was within the range that was reported by Lu et al. (31). The differences observed between the two studies may be explained by differences in genetic background or age of the mice used or differences in study design with the underlying cause likely being multifactorial in nature.

The FEUA presents the ratio between UA and creatinine clearance and highlights the dependence of UA secretion on kidney function. It is well-known that, in patients with gout, FEUA is lower than that in healthy subjects $(11,32)$. Moreover, genetic mutations or polymorphisms in renal and intestinal urate transporters can alter FEUA $(13,33,34)$. FEUA in healthy mice is normally within the range of $5 \%$ to $15 \%$ (23). This means that, in healthy animals, around $90 \%$ of urate that is filtered in the renal proximal tubules is reabsorbed back into circulation. The increased FEUA in URKO mice by $\sim 30 \%$ is a consequence of severe hyperuricemia ( $5 \mathrm{x}$ that of normal) and hyperuricosuria ( $3 \mathrm{x}$ that of normal) due to the lack of hepatic uricase. Following ALLN-346 therapy in the 19-day study, the FEUA was normalized, driven by reductions in both urine and plasma UA concentrations. Interestingly, in the ALLO 90 group, no differences in FEUA were noted between pretreatment and treatment periods despite a reduction in both plasma and urine urate concentrations. Similar results were reported in a human clinical trial in which gout patients treated with allopurinol displayed unchanged FEUA despite a reduction in sUA concentration (11). This result is complicated and may be related to oxypurinol, which acts as a substrate for the URAT1 transporter and competes against urate reabsorption, thereby causing an additional increase in uric acid excretion that can result, as seen in our study, in a small increase or no change in FEUA. Increases in plasma creatinine concentrations were recorded in all groups at the end of the study. The highest plasma creatinine concentration was recorded in the ALLO 90 group. This increased plasma creatinine could offset creatinine clearance in a manner not seen in the other two groups.

To further confirm the mechanism of action of ALLN-346, the concentration of urate was analyzed in chyme samples obtained from various parts of the gastrointestinal tract of the uricase/UrOx deficient mice. A low amount of urate, which was similar between groups, was observed in the digesta obtained from the stomach and colon, which was in agreement with previously published results (35). However, urate levels were several fold higher in the digesta obtained from the small intestine, which is consistent with its role as the primary site of extrarenal urate elimination $(23,36)$. With both ALLN-346 and allopurinol therapies, chyme urate levels were reduced, in line with the reduced plasma urate levels observed; the higher the plasma urate, the higher the urate concentration in the chyme from the small intestine, supporting the notion that the small intestine plays an active role in urate elimination under conditions of severe hyperuricemia.

Unlike existing effective, injectable uricase therapy containing pegloticase, which can cause infusion reactions and anaphylaxis, ALLN-346 is designed specifically as an oral enzyme therapy that acts only within the intestinal lumen, and it is not expected to be absorbed. ALLN-346 is $137 \mathrm{kD}$ in size and, thus, is too large for passive absorption into circulation and with no known mechanism involving receptor binding to facilitate active absorption. Thus, systemic absorption into circulation or possible immunogenicity is not expected. This was indirectly shown in both the 7- and 19-day studies, in which oral therapy with ALLN346 had no adverse effects on mouse well-being and behavior. The mice maintained normal food and water intake as well as body weight, all of which were similar to that observed in the ALLO and control groups. ALLN-346 functions to degrade urate that is secreted from circulation (extrarenal elimination) or that is formed in the intestine itself. It is expected that, over time, oral therapy with ALLN-346 enhances enteric elimination of urate, preventing its reabsorption back into circulation, and thereby reducing serum urate concentrations and overall urate burden to the kidneys and other tissues/organs in patients that have hyperuricemia with gout and/or CKD. ALLN-346 catalyzes the conversion of urate to soluble allantoin, carbon dioxide, and hydrogen peroxide $\left(\mathrm{H}_{2} \mathrm{O}_{2}\right)$. It is expected that soluble allantoin is excreted by the kidneys, and $\mathrm{H}_{2} \mathrm{O}_{2}$ is degraded by catalase present in the intestinal mucosa (37). Also, because ALLN-346 is a protein, it is expected that the majority of the enzyme is excreted via the stool or a portion is digested to peptides and eventually amino acids, similar to food proteins that are subsequently 
reutilized for protein synthesis in the body. Systemic parenteral and subcutaneous administration of recombinant uricases, even those that are PEGylated, profoundly lowers sUA. However, this approach is markedly limited by the immunogenicity of uricases, which are foreign proteins to humans (38). Uricase therapy, exemplified by pegloticase, frequently stimulates development of treatment-emergent antibodies that alter drug pharmacokinetics and, within weeks to months, limit the efficacy of uricase therapy. Whether ALLN-346 is immunogenic like pegloticase and rasburicase and whether potential immunogenicity of ALLN-346 and mucosal immunity processes could impact the long-term efficacy of ALLN-346 remain to be determined in future clinical studies.

Some of the limitations of our studies include that only a single dose of ALLN-346 was tested under variable circumstances with one study providing access to the drug over the entire $24 \mathrm{~h}$ cycle versus in the other, access to the ALLN-346 limited to up to $6 \mathrm{~h}$ at night. Moreover, an initial formulation that was not optimized for gastric survival was utilized in these studies. The described studies were designed as seminal, proof-of-concept, preclinical investigations prior to initiating early clinical trials. Because this concept is novel, it requires initial proof of biologically significant activity. Clearly, the current work demonstrates that machine learning-guided molecular evolution can enable the optimization of uricase stability and activity in the intestinal tract and, when administered as oral enzyme therapy, can reduce both plasma and urine uric acid solely through the degradation of urate that is secreted from circulation or formed in the gut itself. Moreover, there is room for the initial ALLN-346 formulation to be optimized further for human clinical trials to ensure maximal survival of the formulation in the acidic $\mathrm{pH}$ of the stomach. Possible enteric coating of the ALLN-346 in an oral dosage form could maximize the survival of the enzyme prior to it reaching the target site of the small intestine, where extrarenal elimination of urate from circulation is modulated by the ABCG2 and GLUT9 transporters.

The oral therapy with ALLN-346 was well-tolerated throughout the study as evidenced in the animals' normal food and water intake, growth, and behavior. The therapy resulted in significant reduction of plasma urate and normalization of urine uric acid excretion. The effect on plasma urate reduction was similar to that observed following ALLO administration at a dose of $50 \mathrm{mg} / \mathrm{l}$, and the effect on urine uric acid excretion was superior to that observed following administration of the maintenance dose of ALLO at $150 \mathrm{mg} / \mathrm{l}$, which is required

\section{REFERENCES}

1. Khanna D, Fitzgerald JD, Khanna PP, Bae S, Singh MK, Neogi T, et al. American College of Rheumatology guidelines for management of gout. Part 1: systematic nonpharmacologic and pharmacologic therapeutic approaches to hyperuricemia. Arthritis Care Res. (2012) 64:1431-46. doi: 10.1002/acr.21772

2. Zhu Y, Pandya BJ, Choi HK. Prevalence of gout and hyperuricemia in the US general population: the national health and nutrition examination survey 2007-2008. Arthritis Rheum. (2011) 63:3136-41. doi: 10.1002/art.30520 to sustain this animal model. The underlying physiology of hyperuricemia and the extra-renal pathway of uric acid elimination corresponds to the ALLN-346 mechanism of action of the degradation of urate, specifically along the intestinal tract. Thus, ALLN-346 has the potential for use in single or combination drug ULT regimens for treatment of hyperuricemia in gout with and without compromised kidney function.

\section{DATA AVAILABILITY STATEMENT}

All datasets presented in this study are included in the article/supplementary material.

\section{ETHICS STATEMENT}

The animal study was reviewed and approved by University of Lund Ethics Review Committee on Animal Experiments.

\section{AUTHOR CONTRIBUTIONS}

$\mathrm{KP}, \mathrm{AD}, \mathrm{RT}$, ES, and DG were responsible for preparing the manuscript. KP, AD, NM, PS, and SP were involved in performing the study. $\mathrm{KP}, \mathrm{AD}, \mathrm{NM}$, and PS conducted data analyses for the study. AD, RT, SP, and DG designed the study, interpreted the results, and critically reviewed the manuscript. All authors contributed to manuscript revision and have read and approved the submitted version.

\section{FUNDING}

The present study was supported by Allena Pharmaceuticals, USA. RT was funded by the VA Research Service and NIH (AR060772 and AR075990). Both VA Research Service and $\mathrm{NIH}$ were not involved in the study design, collection, analysis, interpretation of data, the writing of this article or the decision to submit it for publication, while Allena Pharmaceuticals was involved in the study design, data analysis, writing of this article and the decision to submit it for publication.

\section{ACKNOWLEDGMENTS}

We thank Dr. Alicia Weeks for critical revision of the manuscript. The authors would also like to express their thanks to Dr. Janine Donaldson for her constructive feedback and valuable input with regards to the writing of the manuscript.
3. Terkeltaub R. Update on gout: new therapeutic strategies and options. Nat Rev Rheumatol. (2010) 6:30-8. doi: 10.1038/nrrheum.2009.236

4. Cicerello E. Uric acid nephrolithiasis: an update. Urologia. (2018) 85:938. doi: $10.1177 / 0391560318766823$

5. Singh JA, Strand V. Gout is associated with more comorbidities, poorer health-related quality of life and higher healthcare utilisation in US veterans. Ann Rheum Dis. (2008) 67:1310-6. doi: 10.1136/ard.2007.081604

6. Jin M, Yang F, Yang I, Yin Y, Luo JJ, Wang H, Yang XF. Uric acid, hyperuricemia and vascular diseases. Front Biosci. (2012) 17:65669. doi: $10.2741 / 3950$ 
7. Stamp LK, Chapman PT. Gout and its comorbidities: implications for therapy. Rheumatology. (2013) 52:34-44. doi: 10.1093/rheumatology/kes211

8. Gustafsson D, Unwin R. The pathophysiology of hyperuricaemia and its possible relationship to cardiovascular disease, morbidity and mortality. BMC Nephrol. (2013) 14:164. doi: 10.1186/1471-2369-14-164

9. Joosten LAB, Crişan TO, Bjornstad P, Johnson RJ. Asymptomatic hyperuricaemia: a silent activator of the innate immune system. Nat Rev Rheumatol. (2020) 16:75-86. doi: 10.1038/s41584-019-0334-3

10. Reginato AM, Mount DB, Yang I, Choi HK. The genetics of hyperuricaemia and gout. Nat Rev Rheumatol. (2012) 8:61021. doi: 10.1038/nrrheum.2012.144

11. Perez-Ruiz F, Calabozo M, Erauskin GG, Ruibal A, Herrero-Beites AM. Renal underexcretion of uric acid is present in patients with apparent high urinary uric acid output. Arthritis Rheum. (2002) 47:610-3. doi: 10.1002/art.10792

12. Sorensen LB. Role of the intestinal tract in the elimination of uric acid. Arthritis Rheum. (1965) 8:694-706. doi: 10.1002/art.1780080429

13. Mandal AK, Mount DB. The molecular physiology of uric acid homeostasis. Annu Rev Physiol. (2015) 77:32345. doi: 10.1146/annurev-physiol-021113-170343

14. Hyndman D, Liu S, Miner JN. Urate handling in the human body. Curr Rheumatol Rep. (2016) 18:34. doi: 10.1007/s11926-016-0587-7

15. Bhatnagar V, Richard EL, Wu W, Nievergelt CM, Lipkowitz MS, Jeff J, Maihofer AX, Nigam SK. Analysis of ABCG2 and other urate transporters in uric acid homeostasis in chronic kidney disease: potential role of remote sensing and signaling. Clin Kidney J. (2016) 9:444-53. doi: 10.1093/ckj/sfw010

16. Dalbeth N, Choi HK, Joosten LAB, Khanna PP, Matsuo H, Perez-Ruiz F, Stamp LK. Gout. Nat Rev Dis Primers. (2019) 5:69. doi: 10.1038/s41572-019-0115-y

17. Garg JP, Chasan-Taber S, Blair A, Plone M, Bommer J, Raggi P, Chertow GM. Effects of sevelamer and calcium-based phosphate binders on uric acid concentrations in patients undergoing hemodialysis: a randomized clinical trial. Arthritis Rheum. (2005) 52:290-5. doi: 10.1002/art.20781

18. Ohno I, Yamaguchi Y, Saikawa H, Uetake D, Hikita M, Okabe H, Ichida $\mathrm{K}$, Hosoya T. Sevelamer decreases serum uric acid concentration through adsorption of uric acid in maintenance hemodialysis patients. Intern Med. (2009) 48:415-20. doi: 10.2169/internalmedicine.48.1817

19. Nigam SK, Bhatnagar V. The systems biology of uric acid transporters: the role of remote sensing and signaling. Curr Opin Nephrol Hypertens. (2018) 27:305-13. doi: 10.1097/MNH.0000000000000427

20. Gutmann H, Hruz P, Zimmermann C, Beglinger C, Drewe J. Distribution of breast cancer resistance protein (BCRP/ABCG2) mRNA expression along the human GI tract. Biochem Pharmacol. (2005) 70:695-9. doi: 10.1016/j.bcp.2005.05.031

21. Nakayama A, Matsuo H, Takada T, Ichida K, Nakamura T, Ikebuchi Y, et al. ABCG2 is a high-capacity urate transporter and its genetic impairment increases serum uric acid levels in humans. Nucleosides Nucleotides Nucleic Acids. (2011) 30:1091-7. doi: 10.1080/15257770.2011.633953

22. Yano H, Tamura Y, Kobayashi K, Tanemoto M, Uchida S. Uric acid transporter ABCG2 is increased in the intestine of the 5/6 nephrectomy rat model of chronic kidney disease. Clin Exp Nephrol. (2014) 18:505. doi: 10.1007/s10157-013-0806-8

23. Takada T, Ichida K, Matsuo H, Nakayama A, Murakami K, Yamanashi $\mathrm{Y}$, et al. ABCG2 dysfunction increases serum uric acid by decreased intestinal urate excretion. Nucleosides Nucleotides Nucleic Acids. (2014) 33:275-81. doi: 10.1080/15257770.2013.854902

24. Matsuo H, Ichida K, Takada T, Nakayama A, Nakashima H, Nakamura T, et al. Common dysfunctional variants in ABCG2 are a major cause of early-onset gout. Sci Rep. (2013) 3:2014. doi: 10.1038/srep02014

25. Woodward OM. ABCG2: the molecular mechanisms of urate secretion and gout. Am J Physiol Renal Physiol. (2015) 309:F4858. doi: 10.1152/ajprenal.00242.2015
26. Vargas-Santos AB, Neogi T. Management of gout and hyperuricemia in CKD. Am J Kidney Dis. (2017) 70:422-39. doi: 10.1053/j.ajkd.2017.01.055

27. Ramirez-Sandoval JC, Madero M. Treatment of hyperuricemia in chronic kidney disease. Contrib Nephrol. (2018) 192:135-46. doi: 10.1159/000484288

28. Wu X, Wakamiya M, Vaishnav S, Geske R, Montgomery C, Jr., Jones P, et al. Hyperuricemia and urate nephropathy in urate oxidase-deficient mice. Proc Natl Acad Sci USA. (1994) 91:742-6. doi: 10.1073/pnas.91.2.742

29. Ichida K, Matsuo H, Takada T, Nakayama A, Murakami K, Shimizu T, Takada Y. Decreased extra-renal urate excretion is a common cause of hyperuricemia. Nat Commun. (2012) 3:764. doi: 10.1038/ncomms1756

30. Sorensen LB, Levinson DJ. Origin and extrarenal elimination of uric acid in man. Nephron. (1975) 14:7-20. doi: 10.1159/000180432

31. Lu J, Hou X, Yuan X, Cui L, Liu Z, Li X, Zhang K. Knockout of the urate oxidase gene provides a stable mouse model of hyperuricemia associated with metabolic disorders. Kidney Int. (2018) 93:69-80. doi: 10.1016/j.kint.2017.04.031

32. Liu S, Perez-Ruiz F, Miner JN. Patients with gout differ from healthy subjects in renal response to changes in serum uric acid. Joint Bone Spine. (2017) 84:183-8. doi: 10.1016/j.jbspin.2016.04.007

33. Tan PK, Miner JN. Uric acid transporter inhibitors for gout. ADMET DMPK. (2017) 5:59-74. doi: 10.5599/admet.5.2.387

34. Stiburkova B, Pavelcova K, Pavlikova M, Ješina P, Pavelka K. The impact of dysfunctional variants of ABCG2 on hyperuricemia and gout I pediatric-onset patients. Arthritis Res Ther. (2019) 21:77. doi: 10.1186/s13075-019-1860-8

35. Yun Y, Yin H, Gao Z, Li Y, Gao T, Duan J, et al. Intestinal tract is an important organ for lowering serum uric acid in rats. PLoS ONE. (2017) 12:e0190194. doi: 10.1371/journal.pone.0190194

36. Preitner F, Bonny O, Laverrière A, Rotman S, Firsov D, Da Costa A, Metref $\mathrm{S}$, Thorens B. Glut9 is a major regulator of urate homeostasis and its genetic inactivation induces hyperuricosuria and urate nephropathy. Proc Natl Acad Sci USA. (2009) 106:15501-6. doi: 10.1073/pnas.0904411106

37. Nemali MR, Usuda N, Kumudavalli Reddy M., Oyasu K, Hashimoto T, Osumi $\mathrm{T}$, et al. Comparison of constitutive and inducible levels of expression of peroxisomal $\beta$-oxidation and catalase genes in liver and extrahepatic tissues of rat. Cancer Res. (1988) 48:5316-24.

38. Guttmann A, Krasnokutsky S, Pillinger MH, Berhanu A. Pegloticase in gout treatment - safety issues, latest evidence and clinical considerations. Ther Adv Drug Saf. (2017) 8:379-88. doi: 10.1177/2042098617727714

Conflict of Interest: RT has a research support from Astra-Zeneca and is consulting to Selecta, Sobi, and Horizon. KP is employed in SGP+Group, Sweden. $\mathrm{AD}$ and DG are employed in Allena Pharmaceuticals, USA and RT served as a paid consultant to Allena Pharmaceuticals, USA. SP is the owner of SGP+Group, Sweden.

The remaining authors declare that the research was conducted in the absence of any commercial or financial relationships that could be construed as a potential conflict of interest.

The authors declare that this study received funding from Allena Pharmaceuticals. The funder was involved in the study design, data analysis, writing of this article and the decision to submit it for publication.

Copyright (C) 2020 Pierzynowska, Deshpande, Mosiichuk, Terkeltaub, Szczurek, Salido, Pierzynowski and Grujic. This is an open-access article distributed under the terms of the Creative Commons Attribution License (CC BY). The use, distribution or reproduction in other forums is permitted, provided the original author(s) and the copyright owner(s) are credited and that the original publication in this journal is cited, in accordance with accepted academic practice. No use, distribution or reproduction is permitted which does not comply with these terms. 